\title{
Influence of Pump-to-Signal RIN Transfer on Noise Figure in Silicon Raman Amplifiers
}

\author{
Xinzhu Sang, Dimitrios Dimitropoulos, Bahram Jalali, Fellow, IEEE, and Ozdal Boyraz, Member, IEEE
}

\begin{abstract}
The effect of the relative intensity noise (RIN), transferred from the pump to the signal, in 1-cm-long chip scale silicon Raman amplifiers is investigated in the presence of nonlinear losses. We show that due to the short waveguide length, the reduction in fluctuations that normally occurs due to "walk-off" between pump and signal waves in fiber amplifiers is inefficient in chip scale Raman amplifiers. In the counterpropagating pump configuration, which leads to minimum frequency RIN transfer, fluctuations up to $1.5 \mathrm{GHz}$ are transferred from the pump to the signal. As a case study, the noise figure degrades by as much as $11 \mathrm{~dB}$ in the silicon waveguide with the free carrier life time of $0.1 \mathrm{~ns}$, when it is pumped with a laser with a RIN value of $-125 \mathrm{~dB} / \mathrm{Hz}$.
\end{abstract}

Index Terms-Amplifier noise, nonlinear optics, Raman scattering, silicon-on-insulator technology.

\section{INTRODUCTION}

$\mathbf{M}$ INIATURIZATION of nonlinear optical devices in highly nonlinear high-index-contrast semiconductor waveguides has attracted considerable attention in recent years. For instance, stimulated Raman scattering has been used for light amplification and lasing in silicon [1]-[6]. Despite their high efficiency, the practical realization of these nonlinear silicon devices requires optimization of power levels to avoid nonlinear losses originating from two-photon absorption (TPA) and TPA-induced free-carrier absorption (FCA) [1], [2], [4]. In addition to efficiency, the noise performance of these devices should be evaluated carefully. Recently, the impact of the nonlinear losses on the noise figure of silicon Raman amplifiers has been investigated [7], and the fluctuation of the pump laser is excluded. Small amplitude perturbations residing on the pump beam characterized as the relative intensity noise (RIN) are transferred to the Stokes wave during Raman amplification. This leads to degradation of the amplifier noise figure. The RIN transfer in fiber Raman amplifiers has been extensively studied previously to assess its impact on optical communication systems [8]. In fibers, the long interaction length and averaging due

Manuscript received July 07, 2008; revised September 01, 2008. First published October 31, 2008; current version published December 12, 2008.

$\mathrm{X}$. Sang is with the Department of Electrical Engineering and Computer Science, University of California, Irvine, CA 92697 USA, and also with the Key Laboratory of Optical and Lightwave Technologies (MOE), Beijing University of Posts and Telecommunications, Beijing 100876, China (e-mail: xzsang@gmail.com).

D. Dimitropoulos and B. Jalali are with the Department of Electrical Engineering, University of California, Los Angeles, CA 90095 USA (e-mail: ddimitr@ee.ucla.edu; jalali@ucla.edu).

O. Boyraz is with the Department of Electrical Engineering and Computer Science, University of California, Irvine, CA 92697 USA (e-mail: oboyraz@ uci. edu).

Color versions of one or more of the figures in this paper are available online at http://ieeexplore.ieee.org.

Digital Object Identifier 10.1109/LPT.2008.2005844 to large group velocity dispersion induced "walk-off" mitigates the RIN transfer at noise frequencies above $10 \mathrm{MHz}$ and $10 \mathrm{kHz}$ in copumped and counterpumped configurations, respectively [8]. However, noise components below the cutoff frequency are amplified and appear as fluctuations in the output signal.

In this letter, we study, for the first time, the pump-to-signal RIN transfer and its contributions to the noise figure in chip scale silicon Raman amplifiers. We show that due to the short waveguide length and small "walk-off" pump fluctuation averaging occurs inefficiently in silicon Raman amplifiers, and RIN frequency components as high as $1.5 \mathrm{GHz}$ are transferred to the signal. This occurs in counterpropagating pump-signal configuration which provides the lowest possible cutoff frequency. For the copropagating case all RIN components of the pump at frequencies of practical interest are transferred to the signal. Nonlinear losses including TPA and FCA influence the $-3 \mathrm{~dB}$ cutoff frequency. As a result, the noise figure of an ideal Raman amplifier degrades as much as $11 \mathrm{~dB}$ when it is pumped with lasers with RIN values equal or greater than $-125 \mathrm{~dB} / \mathrm{Hz}$.

\section{Derivation of Pump to Singal RIN Transfer IN SILICON RAMAN AMPLIFIER}

Noise is associated with photon fluctuations along the gain medium. To estimate the gain evolution and the noise, we should consider nonlinear losses and "walk-off" in our calculations along with the Raman gain. If we assume pump and signal waves traveling in the waveguide with different group velocities, we can write the nonlinear losses and interaction between two waves as follows on a reference plane traveling with the pump wave:

$$
\begin{aligned}
\frac{\partial I_{s}}{\partial z}-d_{ \pm} \frac{\partial I_{s}}{\partial t}= & -\alpha_{s} I_{s}-\alpha_{s}^{F C A} I_{s}-\beta_{T P A} I_{s}^{2} \\
& -2 \beta_{T P A} I_{p \pm} I_{s}+g_{R} I_{p \pm} I_{s} \\
\frac{\partial I_{p \pm}}{\partial z}= & \mp \alpha_{p} I_{p \pm} \mp \alpha_{p}^{F C A} I_{p \pm} \mp \beta_{T P A} I_{p \pm}^{2} \\
& \mp 2 \beta_{T P A} I_{s} I_{p \pm} \mp \frac{\omega_{p}}{\omega_{s}} g_{R} I_{s} I_{p \pm} .
\end{aligned}
$$

The parameters $I_{s, p}$ represent the signal and the pump intensities, $g_{R}$ is the Raman gain coefficient, $\alpha_{\mathrm{s}, \mathrm{p}}$ are the linear loss coefficients, $\omega_{\mathrm{s}, \mathrm{p}}$ are the angular optical frequencies, and $\beta_{T P A}$ is the TPA coefficient. The upper and lower signs of \pm and $\mp$ correspond to the copropagating and counterpropagating configurations, respectively. The TPA-induced free-carrier absorption is given as $\alpha_{j}^{F C A}(z)=1.45 \times 10^{-17}\left(\lambda_{\mathrm{j}} / 1550\right)^{2} N(j=$ $s, p, \lambda_{\mathrm{j}}$ in nanometers) for a steady-state free carrier density of $N(z)=\tau \beta_{T P A} I^{2}(z) /(2 h v)$ (in cubic centimeters) generated by CW pump and Stokes waves [6], where $\tau$ is free-carrier lifetime, and $h v$ is the photon energy. Here total intensity, $I$, is assumed to be $I^{2}(z)=\left(I_{p}+I_{s}\right)^{2}$, including free carriers generated by degenerate and nondegenerate TPA effects. The noise 
figure and the pump to signal RIN transfer is mainly determined by the free carrier loss and the "walk-off" between pump and the signal as: $d_{ \pm}=1 / v_{\mathrm{p}} \mp 1 / v_{\mathrm{s}}$, where $v_{\mathrm{s}}$ and $v_{\mathrm{p}}$ are the group velocities experienced by the Stokes and pump waves in the waveguide. The "walk-off" for the copropagation case is mainly attributed to the group velocity dispersion (GVD) in the waveguide, and it is linearly proportional to local GVD value of $D$ and wavelength separation $\Delta \lambda$ and hence $d_{+} \approx D \Delta \lambda$. In the counterpropagating case, the "walk-off" is mainly due to the opposing velocities and it is estimated to be $d_{-} \approx 2 n_{g} / c$, where $n_{g}$ is the average group index and $c$ is the speed of light in vacuum.

Assuming that the intensity fluctuations have sinusoidal time dependence and the modulation indexes can be separated, we can write the intensity of the pump and Stokes waves at any location along the silicon waveguide as

$$
\begin{aligned}
I_{s}(z, t) & =\bar{I}_{s}(z)[1+M(z, t)] \\
& =\bar{I}_{s}(z)[1+m(z) \exp (i \Omega t)] \\
I_{p \pm}(z, t) & =\bar{I}_{p \pm}(z)\left[1+N_{ \pm}(z, t)\right] \\
& =\bar{I}_{p \pm}(z)\left[1+n_{ \pm}(z) \exp (i \Omega t)\right]
\end{aligned}
$$

where $m(z)$ and $n_{ \pm}(z)$ are the perturbing complex spatial modulation indexes, and $\Omega$ is the angular frequency of the modulation. Substituting (3) and (4) into (1) and (2) we can derive the following differential equations for steady-state optical intensities and the complex spatial modulation indexes:

$$
\begin{aligned}
\frac{\partial \bar{I}_{s}}{\partial z}= & -\alpha_{s} \bar{I}_{s}-\bar{\alpha}_{s}^{F C A} \bar{I}_{s}-\beta_{T P A} \bar{I}_{s}^{2} \\
& -2 \beta_{T P A} \bar{I}_{p \pm} \bar{I}_{s}+g_{R} \bar{I}_{p \pm} \bar{I}_{s} \\
\frac{\partial \bar{I}_{p \pm}}{\partial z}= & \mp \alpha_{p} \bar{I}_{p \pm} \mp \bar{\alpha}_{p}^{F C A} \bar{I}_{p \pm} \mp \beta_{T P A} \bar{I}_{p \pm}^{2} \\
& \mp 2 \beta_{T P A} \bar{I}_{s} \bar{I}_{p \pm} \mp \frac{\omega_{p}}{\omega_{s}} g_{R} \bar{I}_{s} \bar{I}_{p \pm} \\
\frac{d m}{d z}= & i \Omega d_{ \pm} m+\left(g_{R}-2 \beta_{T P A}\right) \bar{I}_{p} n_{ \pm}-\beta_{T P A} \bar{I}_{s} m-\gamma_{s}^{F C A} \\
& \times\left(2 \bar{I}_{s}^{2} m+2 \bar{I}_{p \pm} \bar{I}_{s} m+2 \bar{I}_{p \pm}^{2} n_{ \pm}+2 \bar{I}_{p \pm} \bar{I}_{s} n_{ \pm}\right) \\
\frac{d n_{ \pm}}{d z}= & \mp\left(2 \beta_{T P A}+\frac{\omega_{p}}{\omega_{s}} g_{R}\right) \bar{I}_{s} m \mp \beta_{T P A} \bar{I}_{p} n_{ \pm} \mp \gamma_{p}^{F C A} \\
& \times\left(2 \bar{I}_{s}^{2} m+2 \bar{I}_{p \pm} \bar{I}_{s} m+2 \bar{I}_{p \pm}^{2} n_{ \pm}+2 \bar{I}_{p \pm} \bar{I}_{s} n_{ \pm}\right)
\end{aligned}
$$

where $\gamma_{p, s}^{F C A}=\alpha_{p, s}^{F C A} / I^{2}=1.45 \times 10^{-17}\left(\lambda_{p, s} / 1550\right)^{2}$ $\tau \beta_{T P A} /\left(2 h \nu_{p, s}\right)$. We assume that the pump and signal intensity fluctuations are small, i.e., $|\mathrm{m}|^{2},\left|n_{ \pm}\right|^{2},\left|m \bullet n_{ \pm}\right| \ll 1$, and hence quadratic modulation terms can be neglected. The RIN transfer is calculated based on the ratio of the signal RIN at the waveguide output to the pump RIN at the pump input side [8]

$$
\begin{aligned}
& T_{+}(L, \Omega)=\frac{|M(L, \Omega)|^{2}}{\left|N_{+}(0, \Omega)\right|^{2}} \\
& T_{-}(L, \Omega)=\frac{|M(L, \Omega)|^{2}}{\left|N_{-}(L, \Omega)\right|^{2}} .
\end{aligned}
$$

Equations (5)-(8) can be solved numerically for the spatial evolution of the steady-state intensities and the spatial modulation indexes, and we can obtain RIN transfer functions for copropagating and counterpropagating configurations, respectively.

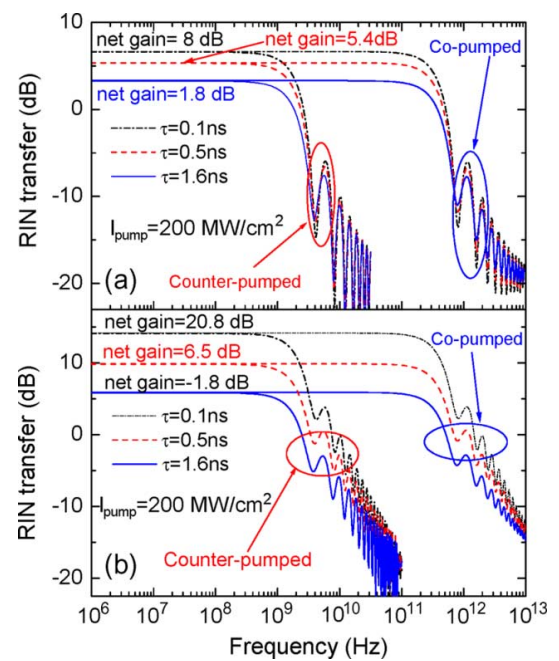

Fig. 1. RIN transfer spectra for copumped and counterpumped silicon Raman amplifiers pumped at (a) $200 \mathrm{MW} / \mathrm{cm}^{2}$ and (b) $10^{3} \mathrm{MW} / \mathrm{cm}^{2}$. Modal parameters: $L=1 \mathrm{~cm}, \alpha_{\mathrm{s}}=\alpha_{\mathrm{p}}=1 \mathrm{~dB} / \mathrm{cm}, g_{R}=15 \mathrm{~cm} / \mathrm{GW}, \beta_{T P A}=$ $0.7 \mathrm{~cm} / \mathrm{GW}, D=-910 \mathrm{ps} /(\mathrm{nm} \bullet \mathrm{km})$, pump at $1418 \mathrm{~nm}$, signal at $1550 \mathrm{~nm}$, and $\tau$ : free carrier lifetime.

\section{RESULTS}

\section{A. Pump to Signal RIN Transfer}

To assess the impact of the RIN transfer, the calculations are performed with waveguide parameters presented in [7] with the pump intensity varying from 10 to $10^{3} \mathrm{MW} / \mathrm{cm}^{2}$. The waveguide length is assumed to be $1 \mathrm{~cm}$ with $1 \mathrm{~dB} / \mathrm{cm}$ linear loss, $g_{R}=15 \mathrm{~cm} / \mathrm{GW}$ Raman gain coefficient and $\beta_{T P A}=0.7 \mathrm{~cm} / \mathrm{GW}$ TPA coefficient. To include the effect of the "walk-off" and GVD, we use GVD parameter of $D=-910 \mathrm{ps} /(\mathrm{nm} \bullet \mathrm{km})$ assuming that the dispersion is mainly determined by the material dispersion and corresponds to the mode area above $1 \mu \mathrm{m}^{2}$ [9]. Additionally, we assume that the Stokes intensity $I_{s}$ is much smaller than the pump intensity $I_{p}$ and depletion of pump due to the Raman gain is negligible.

Fig. 1(a) and (b) shows the magnitude of the pump to signal RIN transfer for pump intensities of 200 and $10^{3} \mathrm{MW} / \mathrm{cm}^{2}$ in a waveguide with free-carrier lifetime values of $0.1,0.5$, and $1.6 \mathrm{~ns}$. The RIN value of the Stokes signal at lower frequencies is expected to be as much as $14.2 \mathrm{~dB}$ worse than that of the pump laser when the net gain is $20.8 \mathrm{~dB}$. Since the RIN transfer depends on the Raman gain, the low frequency RIN transfer decreases noticeably with increasing free-carrier lifetime, i.e., with increasing FCA and decreasing net Raman gain. As it has been observed in fibers, we anticipate that the high-frequency RIN transfer decreases due to the averaging induced by the group velocity difference. However, the $-3-\mathrm{dB}$ cutoff frequency for GVD averaging in a copumped Raman amplifier is $\sim 400 \mathrm{GHz}$. The same value decreases to $\sim 1.5 \mathrm{GHz}$ in a counter pumped Raman amplifier. These values are several orders higher than those expected from fiber Raman amplifiers $(<10 \mathrm{MHz})$. The main reason is that there is not sufficient "walk-off" within a 1-cm-long silicon waveguide to average the effect of the pump intensity noise. Therefore, the RIN transfer noise of silicon Raman amplifiers should be considered even at the high bit rates relevant to the optical communication and the optical signal processing systems. 


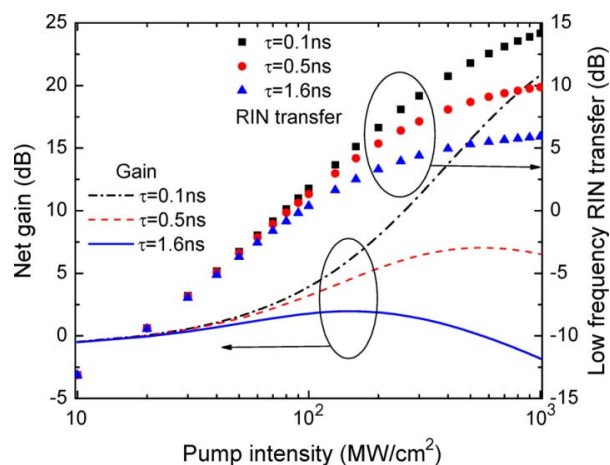

Fig. 2. Low frequency RIN transfer and net Raman gain versus pump intensity. Figure illustrates the impact of the free-carrier lifetime.

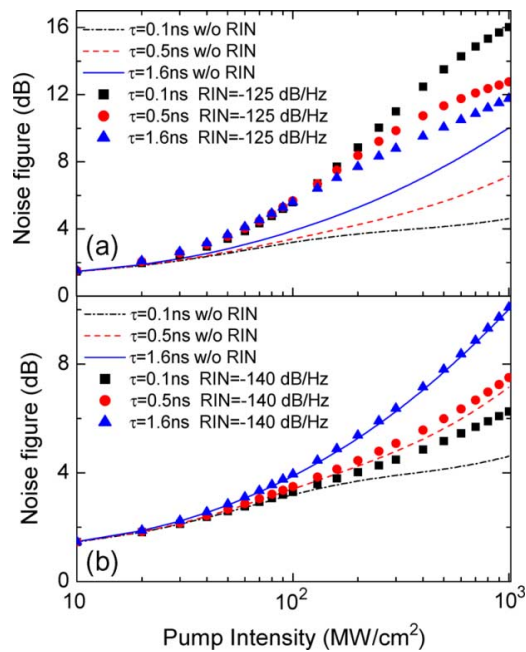

Fig. 3. Noise figure versus pump intensity for (a) pump RIN $=-125 \mathrm{~dB} / \mathrm{Hz}$ and (b) pump RIN $=-140 \mathrm{~dB} / \mathrm{Hz}$.

Fig. 2 illustrates the low-frequency RIN transfer and net Raman gain for pump intensities varying from 10 to $10^{3} \mathrm{MW} / \mathrm{cm}^{2}$. The net Raman gain agrees with the results in [7]. For free-carrier lifetimes above $0.5 \mathrm{~ns}$, the net Raman gain enters into saturation and then decreases with increasing the pump intensity because of nonlinear losses induced by free carriers. However, the RIN transfer is determined by the local gain. This means the pump noise is still transferred into the signal through the stimulated Raman scattering process whether there is net gain amplification at the end or not at high pump intensities.

\section{B. Influence of RIN Transfer on Noise Figure}

In an ideal Raman amplifier, the noise figure is mainly determined by the photon fluctuations along the gain medium and it is estimated to be an increasing value with increasing nonlinear losses [7]. However, typical pump lasers used for Raman amplification are rather noisier than lasers used for optical communication systems ( $>20 \mathrm{~dB}$ worse RIN). Hence, the actual noise figure at the detector should be elevated due to noises transferred from the pump laser and other components. In particular, the final noise figure will increase linearly with increasing RIN transfer rate as $\Delta N F=\Delta R I N \bullet P_{\mathrm{s}, \text { input }} /(2 h v)[10]$. For instance, Fig. 3(a) and (b) illustrates the modified noise figure with respect to varying pump intensities for $-30-\mathrm{dBm}$ weak Stokes wave input. Here, the noise figure is estimated based on the RIN values below cut off frequencies. As expected, the total noise figure increases as much as $11 \mathrm{~dB}$ for a noisy pump laser with $-125 \mathrm{~dB} / \mathrm{Hz}$ RIN value in a Raman amplifier with 20.8 net gain. The noise figure degradation reduces to $2 \mathrm{~dB}$ when a pump laser with $-140 \mathrm{~dB} / \mathrm{Hz}$ is used. For low noisy pump lasers with RIN below $-160 \mathrm{~dB} / \mathrm{Hz}$, the RIN transfer contribution to the noise figure is negligible and we do not expect any measurable degradation.

\section{CONCLUSION}

We show that chip scale Raman amplification in silicon or any other medium will be limited by the noise of the pump lasers. Due to lack of sufficient "walk-off" between the pump and the Stokes waves, the fluctuations on the pump laser will be transferred to the Stokes wave and increase the final noise figure of the amplifier. "Walk-off" induced averaging can be effective for noise frequencies beyond $1.5 \mathrm{GHz}$ if the counterpropagation pumped scheme is implemented. For the copropagating pumping case all the noise components of practical interest will be transferred. As a result, the final noise figure may increase $11 \mathrm{~dB}$ in a 1-cm-long silicon amplifier. Here, we only consider a 1-cm silicon waveguide for compact chip scale devices. Much longer waveguides (up to $8 \mathrm{~cm}$ ) have been demonstrated experimentally for Raman amplification [11], the $-3 \mathrm{~dB}$ cutoff frequencies will decrease to $\sim 49 \mathrm{GHz}$ and $\sim 0.3 \mathrm{GHz}$ for copumped and counterpumped cases, respectively. Long waveguides mean large "walk-off" and low cutoff frequency. For silicon nanowaveguides with the mode area below $1 \mu \mathrm{m}^{2}$, the dispersion characteristics strongly depend on waveguide shape and dimensions [12]. From the noise transfer point of view for Raman amplifiers, waveguide designs with large group velocity dispersion are preferential.

\section{REFERENCES}

[1] O. Boyraz and B. Jalali, "Demonstration of $11 \mathrm{~dB}$ fiber-to-fiber gain in a silicon waveguides," Electron. Expr., vol. 1, pp. 429-434, 2004.

[2] T. K. Liang and H. K. Tsang, "Efficient Raman amplification in silicon-on-insulator waveguides," Appl. Phys. Lett., vol. 85, pp. 3343-3345, 2004.

[3] O. Boyraz and B. Jalali, "Demonstration of a silicon Raman laser," Opt. Express, vol. 12, pp. 5269-5273, 2004.

[4] H. Rong, R. Jones, and A. Liu et al., "A continuous Raman silicon laser," Nature, vol. 433, pp. 725-728, Feb. 2005.

[5] X. Sang, E.-K. Tien, N. S. Yuksek, F. Qian, Q. Song, and O. Boyraz, "Dual-wavelength mode-locked fiber laser with an inctracavity silicon waveguide," IEEE Photon. Technol. Lett., vol. 20, no. 13, pp. 1184-1186, Jul. 1, 2008.

[6] V. M. N. Passaro and F. De Leonardis, "Space-time modelling of Raman pulses in silicon-on-insulator optical waveguides," J. Lightw. Technol., vol. 24, no. 7, pp. 2920-2931, Jul. 2006.

[7] D. Dimitropoulos, D. R. Solli, R. Claps, O. Boyraz, and B. Jalali, "Noise figure of silicon Raman amplifiers," J. Lightw. Technol., vol. 26, no. 7, pp. 847-852, Apr. 1, 2008.

[8] C. R. S. Fludger, V. Handerek, and R. J. Mears, "Pump to signal RIN transfer in Raman fiber amplifiers," J. Lightw. Technol., vol. 19, no. 8, pp. 1140-1148, Aug. 2001.

[9] H. K. Tsang et al., "Optical dispersion, two-photon absorption and selfphase modulation in silicon waveguides at $1.5 \mu \mathrm{m}$ wavelength," Appl. Phys. Lett., vol. 80, pp. 416-418, 2002.

[10] G. Obarski, "Precise calibration for optical amplifier noise figure measurement using the RIN subtraction method," presented at the Optical Fiber Communications Conf., 2003, Paper ThZ3.

[11] V. Sih et al., "Raman amplification of $40 \mathrm{~Gb} / \mathrm{s}$ data in low-loss silicon waveguides," Opt. Express, vol. 15, pp. 357-362, 2007.

[12] E. Dulkeith et al., "Group index and group velocity dispersion in silicon-on-insulator photonic wires," Opt. Express, vol. 14, pp. 3853-3863, 2006. 